\title{
A POTENTIAL EFFECT OF SILVER NANOPARTICLES (Ag-NPs) ON SOME LACTIC ACID BACTERIA GROWTH
}

\author{
El- Fadly, E G; Hassan, N H; Mehanna, N M; and Saleh, Th M* \\ "Dairy Science Department, Faculty of Agriculture, Kafrelsheikh \\ University Egypt
}

\begin{abstract}
This study aimed to essay what could happen if lactic acid bacteria, especially some antifungal lactobacilli strains, mesophilic and thermophilic starter cultures (G+ve model), were treated with Ag-NPs using standard plate count agar assay. As well, E coli (G-ve model) was treated with Ag-NPs in a broth medium. The turbidity was measured for zero, three, six, nine hours and compared to control. The results approved that Ag-NPs had a negligible effect the $\mathrm{G}+\mathrm{ve}$ strains. On the other hand, Ag-NPs had an aggressive activity against the gram-negative one.

Keywords: antifungal Lactobacilli- Ag-NPs - mesophilic and thermophilic starter cultures- Gram positive and negative strains.

\section{1- INTRODUCTION}

Lactobacilli is a main approach under LAB. Lactobacilli strains have employed not only as starter cultures with probiotic functions but also as an antifungal agent. Recently, another consideration took place where silver nanoparticles (Ag-NPs) were revived to be used as an antimicrobial agent (Kim et al., 2007). This approach had a worldwide acceptance because $\mathrm{Ag}$ and its compounds were famed as non-toxic, undercover inorganic efficient antimicrobial agents, which can control the growth of about 650 type of microorganisms (Jeong et al., 2005). Notably, silver nanoparticles (Ag-NPs) drew attention as a potential antimicrobial (fungal, and bacterial) agent. This interest was due to the fast progress in research on metal nanoparticles (NPs) and the potential superiority of nano size antimicrobial activity compared to bulk silver metals. Also, Ag-NPs have higher specific surface area and high surface atoms fraction (Kim et al., 2009; Lee, et al., 2009 and Noorbakhsh et al., 2011). Therefore, Ag-NPs present a very promising future to solve one of the biggest problems in dairying, which is the growth of foodborne fungi (Plocková, et al., 2001; Oliveira et al, 2014; Medina and Jordano 1993 and Banjara et al., 2015). In this context, it was necessary to investigate the effect of the presence of Ag-NPs as a novel antifungal agent on some individual lactobacilli strains and starter cultures blends, on the other hand.
\end{abstract}




\section{1- Microorganisms:}

\section{2- MATERIALS AND METHODS}

\subsection{G+ve bacteria:}

1.1.1. Lactobacilli strains:

Lb. casei NRRL B-441 (Lb. casel) (4) Lb. delbruckii NRRL B1024 (Lb. delbruckii) (5) Lb. plantarum NRRL B-531 (Lb. plantarum) (9) U.V. mutant from original strain $L b$. casei ssp. rhamnosus NRRL B445 (Lb. rhamnosus uv mutant) (10) were Supplied as a gift from $\operatorname{Dr} A$. A. Khattab (National Research Center, Gizza, Egypt).

\subsubsection{Starter culture blends:}

Two freeze-dried lactic cultures for Direct Vat Set (DVS) were used. Mesophilic/ thermophilic culture blend (Chr-Hansen FRC-60) containing Lactococcus lactic, Lactococcus cremoris, Streptococcus thermophilus, and lactobacillus bulgaricus. Thermophilic culture (ChrHansen TTC-3) containing Streptococcus thermophilus, and lactobacillus bulgaricus.

\subsection{G-ve bacteria:}

Two pathogenic bacterial strains $E$ coli 076 (G-ve). were a gift from the central laboratory for Microbiology, Faculty of Veterinary Medicine, Kafrelsheikh University Egypt.

\section{2- Materials:}

Silver nanoparticles were prepared, characterised as described by Kim et al., 2007. (8-11nm) particles suspension with a concentration of $(1000 \mu \mathrm{l} / \mathrm{ml})$ was used in this experiment. A 10000 $\mu \mathrm{l} / \mathrm{ml}$ sterilized stock suspension was employed.

\section{3- Microbiological assays:}

\subsection{Ag-NPs effect on the lactic acid bacterial growth:}

\subsubsection{The effect on lactobacilli strains:}

The standard plate count assay was carried out. Where a standard freshly prepared MRS agar medium was mixed with $(10 \mathrm{ml}$ the stock Ag-NPs suspension/100ml medium) just before pouring in the Petri dishes. Each plate contained $15 \mathrm{ml}$ with the previous mixture mixed with an individual lactobacilli strain. The same experiment was carried out without adding Ag-NPs to the growth medium as the control negative. All plates were incubated at $37^{\circ} \mathrm{C} / 72 \mathrm{~h}$. This assay was carried out in three replicates for each strain. The final results were expressed as log CFU for each strain compared to the control plates.

\subsubsection{The effect on starter cultures blends:}

To evaluate the effect of Ag-NPs on the growth of the thermophilic and mesophilic lactic acid bacterial strains, Bromo cresol green whey agar (BGWA) medium was employed. This medium could 
be used to differentiate, and consequently enumerate Streptococcus thermophiles and Lactobacillus bulgaricus individually on the same plate as described by Yamani and Ibrahim 1996. The Streptococcus thermophillus colonies were green lenticular with entire edges, while Lactobacillus bulgaricus colonies were larger and mostly of light color with greenish centres, irregular in shape and in the form of a mass with twisted or fuzzy filament projections. The same previously mentioned method in 3.1.1. was carried out.

\subsection{The effect of Ag-NPs on E coli growth rate:}

To study the Ag-NPs effect on the selected G-ve bacterial strain, three $100 \mathrm{ml}$ conical flasks containing $50 \mathrm{ml}$ of Macconkey broth medium (Oxoid) were used. Two of them were inoculated with approximately $1 \times 10^{6}$ CFU of freshly prepared broth of $E$. coli (Agnihotri et al., 2014), while the third did not inoculate. The Ag-NPs suspension was added to the first one $(\mathrm{Ag})$ and the final concentration was adjusted to $1000 \mathrm{ppm}$. In parallel, the second flask was considered a control (C) and the third was a blank (B). Then, the three flasks were incubated in a shaker incubator (Temperaturaturbegrenzer, 3022, Germany (150 rpm) at $37{ }^{\circ} \mathrm{C} / 9 \mathrm{hr}$. Growth was measured at three hours intervals by monitoring the optical density at absorbance $600 \mathrm{~nm}$ for $E$. coli using spectrophotometer (UV-1800 SPECTROPHOTOMETER) according to Quigley 2008. In parallel, the bacterial count was also determined using standard plate count technique in Macconkey agar media incubated at $37^{\circ} \mathrm{C}$ for $24 \mathrm{hr}$. The two measurements were plotted together, and a growth curve was obtained for the two strains to estimate the log bacterial count (log CFU) correspondence to the absorbance. This trial was carried out in three typical replicates, and only one curve from each group was taken into consideration.

\section{Statistical analysis:}

Data from three independent replicate experiments analyzed using Statistical Package for the Social Sciences (SPSS) 16.0 statistical software (Chicago, USA). The data are reported as the mean \pm standard error. Significant differences between mean values were located with T-test and Duncan's Multiple Range test $(p<0.05)$, followed by one-way ANOVA.

\section{RESULTS AND DISCUSSION}

1- Silver nanoparticles (Ag-NPs) potential effect on LAB:

\subsection{Ag-NPs effect on antifungal lactobacilli individual strains:}

Figure 1 illustrates the Ag-NPs effect on the lactobacilli colonies counts compared to control. The histograms show that there is no significant difference between the strains that had been inoculated into 
MRS agar medium containing Ag-NPs when compared to the same strains in MRS agar medium only.

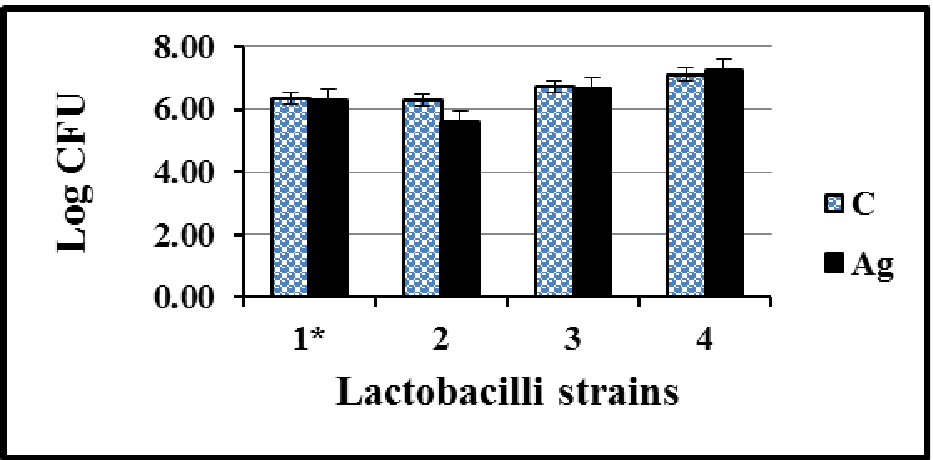

Fig (1): lactobacilli logarithmic colonies count in MRS agar medium with(Ag) and without (C) Ag-NPs after incubation at $\left(37^{\circ} \mathrm{C} / 72 \mathrm{~h}\right)$.

"(1) Lb. casei NRRL B-441, (2) Lb. delbrueckii NRRL B-1024, (3) Lb. plantarum NRRL B-531, (4) U.V. mutant of original strain $L b$. casei ssp. rhamnosus NRRL B-445.

a,b,c, Means with unlike small superscript letters within the same column group, are significantly different $(P<0.05)$.

\subsection{Ag-NPs effect on starter cultures blends:}

Both thermophilic (TTC-3) and mesophilic (FRC-60) starter cultures had the same previous trend of the individual lactobacilli strains. Where shown in fig (2) there is no significant difference between colonies count in control medium compared to the same starter cultures counts in the medium containing Ag-NPs.

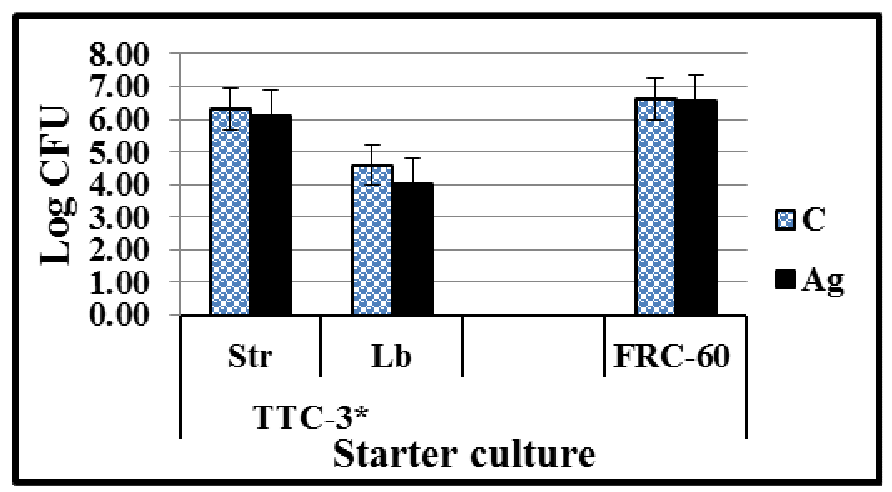

Fig (2) Starter cultures logarithmic colonies count in (BGWA) agar medium with $(\mathrm{Ag})$ and without $(\mathrm{C}) \mathrm{Ag}-\mathrm{NPs}$ after incubation at $\left(37^{\circ} \mathrm{C} / 72 \mathrm{~h}\right)$.

"(TT-3) thermophilic starter culture containing: ( Str: Str thermophillus and Lb: Lb bulgaricus) and (FRC-60) mesophilic starter culture.

$a, b, c$, Means with unlike small superscript letters within the same column group, are significantly different $(P<0.05)$. 


\section{The effect Ag-NPs on $E$ coli growth rate:}

Fig (3) is an attempt to elucidate the extent of the bactericidal effect of Ag-NPs concentration on the pathogenic bacteria viability. $E$ coli as the Gram-negative "G-ve" model was examined. Fig 3 (A) reveals the growth profile of the strain in the control media (growth media without Ag-NPs). While figure (B) shows that Ag-NPs had a remarkable effect on the G-ve strain viability. $E$. coli had a different trend comparing to the control. Ag-NPs presence led to growth rate rapid decrement.

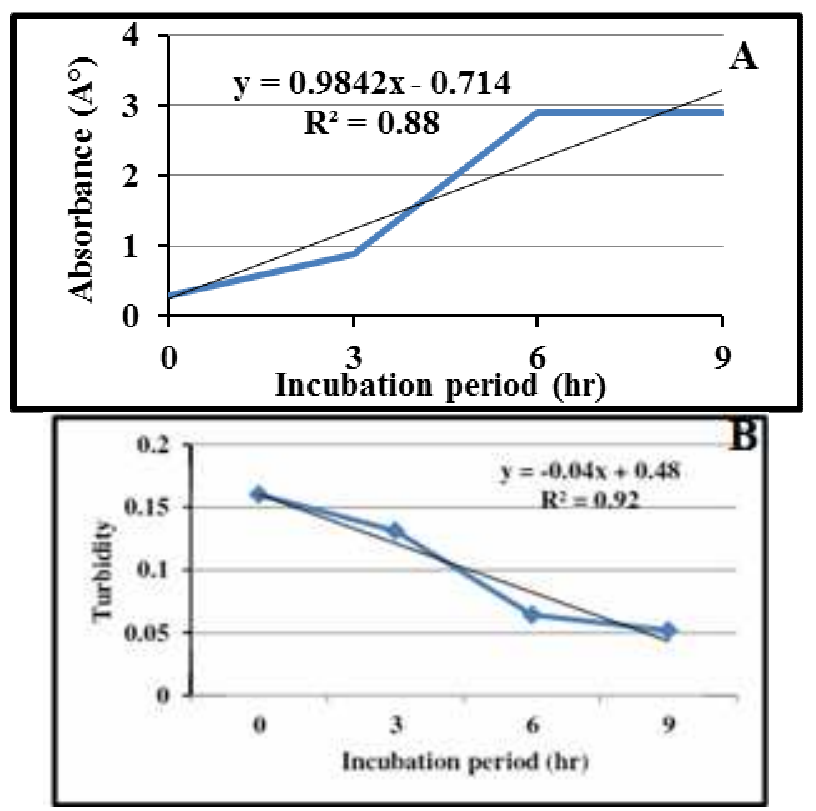

Fig (3): Growth profiles of $E$ coli 076. (A) control and (B) in the presence of $1000 \mathrm{ppm}$ of $\mathrm{Ag}-\mathrm{NPs}$, for $\left(9 \mathrm{hr} / 37^{\circ} \mathrm{C}\right)$

Fig (4) elucidates the media color changes compared to the blank (B). Bacterial strain growth causes medium turbidity in the control (C). Ag-NPs treatment (Ag) got a darker color as a result of silver particles present but didn't show any bacterial growth indicators in Macconkey broth containing $E$ coli initial viable cells as much as the control medium. 


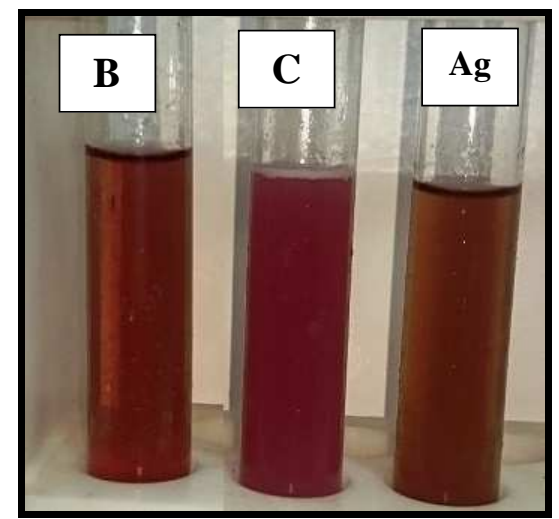

Fig (4): Macconkey broth medium after inoculation with $E$ coli 076 (B) blank media, (C) control, and $(\mathrm{Ag})$ in the presence of $1000 \mathrm{ppm}$ of $\mathrm{Ag}-\mathrm{NPs}$. All tubes were incubated for $\left(9 \mathrm{hr} / 37^{\circ} \mathrm{C}\right)$

Aiming to discuss these results, silver ions are well-known to be effective against a broad variety of microorganisms, including sixteen major bacterial species. This feature makes silver an important choice for the multi-resisting role in food industry field. In the same context, Ag-NPs showed the same behaviour but in tougher ways. This activity is due to the particles huge surface area that the microorganisms expose to. Though Ag-NPs have so many antibacterial applications in different fields, silver nanoparticles antibacterial mode of action is not completely understood (Prabhu and Poulose 2012). In general, low concentrations of Ag-NPs as one of the heavy metals can motivate significant changes in cell functions and structure thus the microorganisms could be destroyed. When used at high concentrations, Ag-NPs behave as a protoplasmic poison; that denature proteins and nucleic acid (Ogar et al., 2015).

In light of these hypotheses, Ag-NPs mode of action could be explained for several prospects. From a morphological standpoint, AgNPs have a dimension ranges from (1-100) $\mathrm{nm}$. As a result of this tiny size, Ag-NPs have a huge surface area /volume ratio. So, as mentioned, Ag-NPs have unique physical, chemical, biological characteristics compared to their bulk origin. Thereby, Ag-NPs can physically interact with several bacteria cell surface. This anticipation is important, particularly when discussing Ag-NPs antibacterial activity against $E$ coli, where Ag-NPs adhere and accumulate on the bacterial surface. This physical contact leads nanoparticles to attach to lipopolysaccharides layer in cell membranes causing structural changes which in turn make bacterial membrane more permeable. For more elucidation, Ag-NPs direct interaction creates gaps in cell 
membrane bilayer causing excessive permeability that leads finally to cell death (Franci et al., 2015).

In our experiment, $E$ coli had unusual growth profile when subjected to Ag-NPs compared to the negative control. Where the growth curve showed that it had a slow turbidity decrement during the first $3 \mathrm{hr}$, then it dropped in a fast rate indicating cells death. This growth pattern may be briefed in two explanations: first; Ag-NPs have the ability to interact with various microorganisms (such as bacteria) and also impact both the growth of and mature bacterial biofilms and, therefore, could be used as broad spectrum antimicrobials. The antibacterial effect appears to be conferred by their ultra small size and increased surface area, through which they destroy the membrane, cross the body of the microbe and create intracellular damage. Due to the structural difference in the composition of the cell walls of $G+v e$ and G-ve Ag-NPs have significantly less effect on the growth of $G+v e$ bacteria. The Gram-negative bacteria have a layer of lipopolysaccharides on the outside and present below a thin $(7$ to 8 nanometers) layer of peptidoglycan. Although lipopolysaccharides are composed of lipids covalently bound to polysaccharides, there is a lack of rigidity of the overall structural envelope. The negative charges on the lipopolysaccharides are attracted to the weak positive charge of Ag-NPs. On the other hand, the cell wall of G+ve bacteria ( $L A B$ are a clear example) is mainly composed of a thick layer (20 to 80 nanometers) of peptidoglycan consisting of linear polysaccharidic chains cross-linked by short peptides to form a three-dimensional rigid structure. The stiffness and the extensive cross-linking not only reduce the bacterial cell wall anchoring sites for Ag-NPs but also render the wall itself more difficult to penetrate (Franci et al., 2015).

The second explanation is helping to understand $E$ coli unusual growth rate as it is related to the cells age, according to the fact that $E$ coli generation time ranges between 27-30 min. Where at the first $3 \mathrm{hr}$ of incubation, the domain cells are the mother cells where cell membrane is thicker and harder to be affected by the Ag-NPs presence. So it needs much more time to be damaged. As time goes by, each mother cell divides into two daughter cells where they are weaker and easier to be affected by the nanoparticles exposure. Returning to the antibacterial Ag-NPs mode of action possibilities, another point of view is that the Ag-NPs render a permanent damage on bacterial cells by inhibiting DNA replication, modifying intracellular ATP levels and damaging cytoplasm membranes. Theories explaining this depend on a hypothesis that Ag-NPs can release silver ions and free radicles. Silver ions interact with the thiol groups $(-\mathrm{SH})$, which are a constituent of many vital enzymes, leading to blocking them. Therefore, several functions will be inhibited causing irreversible cell 
damage. One of these inhibited functions is suppressing the respiratory enzymes causing generating reactive oxygen species that attack the cell itself. Also, citrate-capped Ag-NPs tend to be a weak acid which in turn tends to react with weak bases. Sulfur and phosphorus are weak bases, and they are the major cell components. Consequently, the reaction among these elements and Ag-NPs in the cell causes its death. In the same context, DNA major components are sulphur and phosphate, which explain why Ag-NPs complex with it; causing replication problems and eventually terminating the microbe (Agnihotri et al., 2014; Prabhu and Poulose 2012).

These approaches may have occurred in parallel which elucidates the G-ve quick response towards Ag-NPs. The Ag-NPs antibacterial activity results in this work go with the previous studies work (Kim et al., 2007; Agnihotri et al., 2014; Franci et al., 2015). On the other hands, others confirmed that Ag-NPs had a remarkable inhibiting effect on both $\mathrm{G}-\mathrm{ve}$ and $\mathrm{G}+\mathrm{ve}$ strains. This contradiction may also retune to the fact that the antibacterial mechanism(s) of Ag-NPs is not entirely recognized (Devi and Bhimba 2014; Noorbakhsh et al., 2011). Therefore, Ag-NPs antibacterial activity against G-ve and G+ve bacteria needs more profound investigations.

\section{REFERENCES}

Agnihotri, S., Mukherji, S. and Mukherji, S. (2014) 'Size-controlled silver nanoparticles synthesized over the range 5-100 nm using the same protocol and their antibacterial efficacy', RSC Adv., 4(8), pp. 3974-3983. doi: 10.1039/C3RA44507K.

Banjara, N., Suhr, M. J. and Hallen-adams, H. E. (2015) 'Diversity of yeast and mold species from a variety of cheese types', Curr Microbiol, 70, pp. 792-800. doi: 10.1007/s00284-015-0790-1.

Chudobova, D., Dobes, J., Nejdl, L., Maskova, D., Rodrigo, M. A. M., Nedecky, B. R., Krystofova, O., Kynicky, J., Konecna, M., Pohanka, M., Hubalek, J., Zehnalek, J., Klejdus, B., Kizek, R. and Adam, V. (2013) 'Oxidative stress in Staphylococcus aureus treated with silver(i) ions revealed by spectrometric and voltammetric assays', International Journal of Electrochemical Science, 8(4), pp. 44224440.

Devi, J. S. and Bhimba, B. V. (2014) 'Antibacterial and antifungal activity of silver nanoparticles synthesized using Hypnea muciformis', Biosciences Biotechnology Research Asia, 11(1), pp. 235-238. doi: $10.13005 / \mathrm{bbra} / 1260$.

Franci, G., Falanga, A., Galdiero, S., Palomba, L., Rai, M., Morelli, G. and Galdiero, M. (2015) 'Silver Nanoparticles as Potential Antibacterial Agents', Molecules, 20(5), pp. 8856-8874. doi: 10.3390/molecules20058856.

Jeong, S. H., Yeo, S. Y. and Yi, S. C. (2005) 'The effect of filler particle size on the antibacterial properties of compounded polymer/silver 
fibers', Journal of Materials Science, 40(20), pp. 5407-5411. doi: 10.1007/s10853-005-4339-8.

Kim, J. S. J.-H., Kuk, E., Yu, K. N., Kim, J. S. J.-H., Park, S. J., Lee, H. J., Kim, S. H., Park, Y. K. Y. H., Park, Y. K. Y. H., Hwang, C.-Y., Kim, Y.K., Lee, Y.-S., Jeong, D. H. and Cho, M.-H. (2007) 'Antimicrobial effects of silver nanoparticles.', Nanomedicine : nanotechnology, biology, and medicine, 3(1), pp. 95-101. doi: 10.1016/j.nano.2006.12.001.

Kim, K.-J., Sung, W. S., Suh, B. K., Moon, S.-K., Choi, J.-S., Kim, J. G. and Lee, D. G. (2009) 'Antifungal activity and mode of action of silver nano-particles on Candida albicans.', Biometals: an international journal on the role of metal ions in biology, biochemistry, and medicine, 22(2), pp. 235-242. doi: 10.1007/s10534-008-9159-2.

Lee, J., Kim, K-J., Sung, W.S., Kim, J. G. \& Lee, D. G. (2009) 'The Silver Nanoparticle ( Nano-Ag ): a New Model for Antifungal Agents', Silver Nanoparticles, pp. 295-309.

Medina, L. M. and Jordano, R. (1993) 'Growth of Fungal Contamination in Fermented Milk Containing Bifidobacteria And Lactobacillus addophttus', Journal of Food Quality, 16(6), pp. 471-477. doi: 10.1111/j.1745-4557.1993.tb00272.x.

Noorbakhsh, F., Rezaie, S. and Shahverdi, A. R. (2011) 'Antifungal Effects of Silver Nanoparticle alone and with Combination of Antifungal Drug on Dermatophyte Pathogen Trichophyton Rubrum', 2011 International Conference on Bioscience, Biochemistry and Bioinformatics., 5, pp. 364-367.

Ogar, A., Tylko, G. and Turnau, K. (2015) 'Antifungal properties of silver nanoparticles against indoor mould growth', Science of the Total Environment. Elsevier B.V., 521-522, pp. 305-314. doi: 10.1016/j.scitotenv.2015.03.101.

Oliveira, P. M., Zannini, E. and Arendt, E. K. (2014) 'Cereal fungal infection, mycotoxins, and lactic acid bacteria mediated bioprotection: from crop farming to cereal products.', Food microbiology, 37, pp. 78-95. doi: 10.1016/j.fm.2013.06.003.

Plocková, M; Stiles, J; Chumchalová, J. and H. R. (2001) Control of mould growth by Lactobacillus rhamnosus VT1 and Lactobacillus reutreri CCM 3625 on milk agar plates, Czech J Food Sci.

Prabhu, S. and Poulose, E. K. (2012) 'Silver nanoparticles: mechanism of antimicrobial action, synthesis, medical applications, and toxicity effects', International Nano Letters, 2(1), p. 32. doi: 10.1186/22285326-2-32.

Quigley, T. (2008) 'Monitoring the Growth of E . coli With Light Scattering Using the Synergy ${ }^{\mathrm{TM}} 4$ Multi-Mode Microplate Reader with Hybrid Technology TM, BioTek Application Note, (3603).

Yamani, M. I. and Ibrahim, S. A. (1996) 'The differential enumeration of Lactobacillus delbrueckii subspecies bulgaricus and Streptococcus salivarius subspecies thermophilus in yogurt and labneh using an improved whey medium', International Journal of 
Dairy Technology, 49(4), pp. 103-108. doi: 10.1111/j.14710307.1996.tb02500.x.

$$
\text { الملخص العربي }
$$

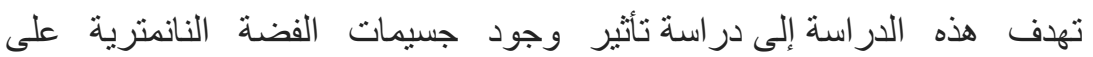

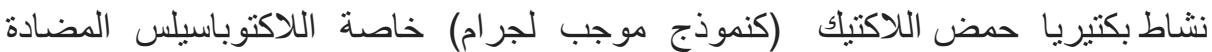

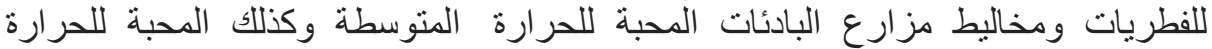

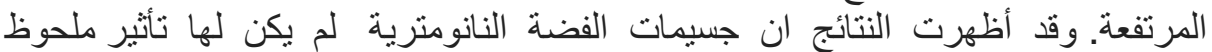

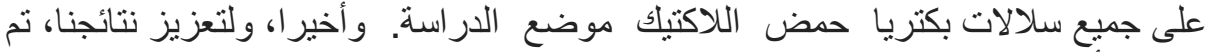

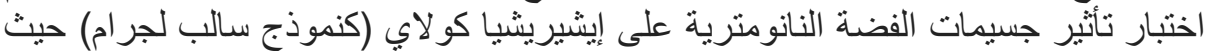

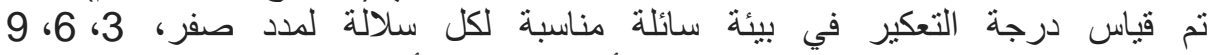

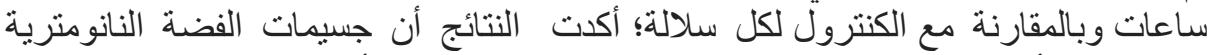

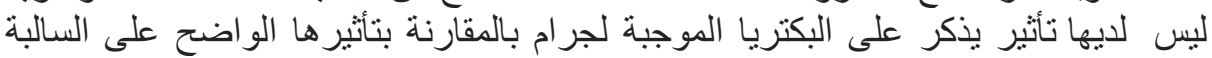
لجرام. 